\title{
A Novel Approach for Determining Permeability in Porous Media Aminu $\mathrm{MD}^{1 *}$ and Ardo $\mathrm{BU}^{2}$
}

${ }^{1}$ Department of Offshore, Process and Energy, School of Energy, Environment and Agrifood, Cranfield University, Bedfordshire, MK43 0AL, UK ${ }^{2}$ Department of Geology, Modibbo Adama University of Technology, Yola, PMB 2076, Adamawa State, Nigeria

\begin{abstract}
Permeability can be grouped into three different types; absolute permeability, relative permeability, and effective permeability Rocks have different permeability properties; some rocks permit the flow of fluids through its pores effectively, others only do so nominally. The permeability of rocks equally relates to the rock's capacity to allow the flow of petroleum, water and gas through it. Unlike porosity, which is a function of the volume of voids over the total volume of rock fabric (usually expressed in percentage), permeability concentrates only in the simplicity that petroleum encounters as it flows between interconnected rock pores. In real life scenarios however, because of the multiphase flow (that is because not only one fluid would flow between the interconnected rock pores), different types of permeability can be encountered such as absolute permeability, relative permeability and effective permeability. In part A of this paper, absolute permeability is what is in study because it is the permeability that is dependent on reservoir rock, not taking into cognizance, the compressibility of fluid (liquid) that flows through the rock. Effective permeability is a measure of the ability of rock particle distribution and its function to allow the flow of petroleum due to different shapes that makes the rock fabric material. Finally, relative permeability describes the percentage of fluids flowing in a competitive multiple phase flow as compared to the flow of the same fluid at 100 percent saturation.
\end{abstract}

Keywords: Permeability; Porous media; Pressure

\section{Nomenclature}

$\begin{array}{lll}\text { Symbol } & \text { Meaning } & \text { Units } \\ \mathrm{K} & \text { Permeability } & (\text { Darcy }) \\ \mathrm{Q} & \text { volumetric flow rate } & \left.\mathrm{m}^{3} \mathrm{~s}^{-1}\right) \\ \mu & \text { Viscosity } & (\mathrm{cp}) \\ \mathrm{L} & \text { length of bed } & (\mathrm{m}) \\ \mathrm{A} & \text { cross sectional area of bed } & \left(\mathrm{m}^{2}\right) \\ \Delta \mathrm{P} & \text { flow rate of phase } & (\mathrm{atm}) \\ \mathrm{Qi} & \text { flow rate of phase } & \mathrm{I} \\ \mathrm{Kri} & \text { relative permeability of phase /fluid } & \mathrm{I} \\ \mathrm{G} & \text { gravity } & \left(\mathrm{ms}^{-1}\right) \\ \mathrm{P} & \text { density } & \left(\mathrm{kg} \mathrm{m}^{-3}\right)\end{array}$

\section{Introduction}

The main objective of this experiment is to determine the absolute permeability of sand as a porous media. Permeability $(K)$ can be described as the ability of a rock to allow the flow of fluids, that is, oil, gas, or water. Then concept of permeability is very important in the petroleum industry in that it is applicable in the measure of resistance of a fluid that flows through it. The key results are shown in Table 1. The word "porous media" in this context is connected with sand because this work in question was carried out with sand as permeable material. Normally, permeability like this is referred to as absolute for the reason that regardless of the working fluid, $\mathrm{K}$ depends on the structure of the porous medium alone. Darcy's law defines the equation of permeability in terms of measurable quantities. There are four conditions necessary for Darcy's equation to be acceptable [1]. These are:

- $\quad$ Single phase flow

- The porous material must not react with the working fluid

- No accumulation
- Creeping flow - the Reynolds' number based on superficial velocity must be on the order of 1 .

$Q \Rightarrow \frac{A K \Delta P}{\mu L}$

Where:

$\mathrm{K}=$ permeability

$\mathrm{Q}=$ volumetric flowrate

$\mu=$ viscosity

$\mathrm{L}=$ length of bed

$\mathrm{A}=$ cross sectional area of bed

$\Delta \mathrm{P}=$ pressure drop

However in the real life scenario it's slightly different because the flow is normally in multiphase flow instead of single phase because the engineers have to account for others fluids to be present in the porous media, e.g. water and oil flowing at the same time through porous rock.

For this reason relative permeability $(\mathrm{Kr})$ is the new quantity that has to be taken in consideration due to microscopic interactions between the liquids, where local fluctuations could be caused in the pressure gradient across the sample.

Therefore relative permeability $(\mathrm{Kr})$ is a quantity that will account for each of the fluid present in the eventual multiphase flow, and the extent to which the fluids hinder one another. Relative permeability therefore will be related to the new (non-compressible) fluid flowing in

*Corresponding author: Aminu MD, Department of Offshore, Process and Energy, School of Energy, Environment and Agrifood, Cranfield University, Bedfordshire MK430AL, UK, Tel: 44-1234-750111; E-mail: m.aminu@cranfield.ac.uk

Received April 18, 2015; Accepted May 06, 2015; Published May 13, 2015

Citation: Aminu MD, Ardo BU (2015) A Novel Approach for Determining Permeability in Porous Media. J Pet Environ Biotechnol 6: 226. doi:10.4172/2157-7463.1000226

Copyright: $\odot 2015$ Aminu MD, et al. This is an open-access article distributed under the terms of the Creative Commons Attribution License, which permits unrestricted use, distribution, and reproduction in any medium, provided the original author and source are credited. 
Citation: Aminu MD, Ardo BU (2015) A Novel Approach for Determining Permeability in Porous Media. J Pet Environ Biotechnol 6: 226. doi:10.4172/2157-7463.1000226

\begin{tabular}{|c|c|}
\hline Area of bed $\left(\mathrm{cm}^{2}\right)$ & 11.34 \\
\hline Length of bed $\left(\mathrm{cm}^{2}\right)$ & 30.5 \\
\hline Average temperature $\left({ }^{\circ} \mathrm{C}\right)$ & 21.5 \\
\hline viscosity of water $(\mathrm{cp})$ & 1115.6 \\
\hline gradient of graph & 237.21433 \\
\hline Permeability (Darcy) & $637.9456577 \mathrm{D}$ \\
\hline
\end{tabular}

Table 1: Key results.

the same rock. The rock permeability does not change, but the flow rate of the new fluid and the equivalent pressure gradient will be accounted, and described as:

$$
\text { Relative.permeability } \Rightarrow \frac{\text { flowrate.multiphase.flow }}{\text { Flowrate.fluid.at.100\%.saturation }} * 100
$$

For this reason the Darcy's law has to be rewritten to account for each fluid, and the middle term of the equation below is referred to as the "mobility" of phase/fluid i and Kkri represents the total permeability of phase $i$ as:

$$
Q i \Rightarrow\left(\frac{K k r i}{\mu i}\right) \frac{A \triangle P i}{L}
$$

Where:

$\mathrm{K}=\mathrm{absolute}$ permeability of medium

$\mathrm{Q} i=$ flow rate of phase $\mathrm{i}$

$\mu_{i}=$ the viscosity of phase i

$\mathrm{L}=$ length of medium

$\mathrm{A}=$ cross sectional area of medium

$\Delta \mathrm{P}=$ pressure drop

However permeability of the rock (sand) in this case could be affected by different factors, where some of these factors listed below are the ones which more affect the permeability of a porous media:

1. Particle size distribution

2. Voids/porosity

3. The degree of saturation

4. Temperature

\section{Where:}

Particle size distribution, relates the size of the porous space, therefore this size will be dependent on the particle size, whereas if the particles are small the size between them will be small resulting in a decrease of permeability because the flow of the fluids will depend on the ability of the rock pore space to allow the flow [2].

In the other hand if the particles are bigger the space between the pores will be bigger resulting in an increase in permeability.

Porosity, relates the degree of compaction of the particle or how more or less compact are the particles because the matrix of this particle distribution will affect as well on the size of the voids between the particles.

The degree of saturation relates the percentage of water filling the voids spaces as it will affect the permeability results, because the blockage of the pores by air bubbles can reduce the permeability considerably.

This way if the degree of saturation is less than $85 \%$, air is likely to be continuous instead of being isolated bubbles, which invalidates Darcy's law", this way is very important to eliminate air bubbles from the matrix.

Temperature, relates that the permeability will increase when the temperature increases, because the viscosity of the water decreases with the rise of temperature and vice versa, therefore is important to take note of water temperature [3].

\section{Description Of Experimental Procedure}

- The pump was switched on after all the valves were kept closed

- After the experiment was started when a constant water level in the overhead tank indicated a constant trickle from the overflow pipe

- Valves (1 to 4 ) were kept closed in order to open the manometer valves (5 to 8 )

- To collect any of the bed material that passed through the sieve and preventing it from returning to the system the drain tube from valve 4 was inserted into a beaker placed on the water tank

- To get the water temperature a thermometer was also placed in the beaker

- After the height of the bed was recorded (305) as well the water and mercury manometer zero levels

- For the water go through the column in a down flow direction valves 1 and 4 were open

- The flow rate $(\mathrm{Q})$ was adjusted using valve 1 where the starting point was $50 \mathrm{~cm}^{3}$, and the various flow rates were noted at the different manometer levels.(Attention was being made to close valves 5 and 6 in case the levels in the water manometer exceeded the scale limits, to shut off the water manometer, it didn't happen)

- Finally to get the average water temperature, the water temperature was taken regularly during the proceeding

\section{Calculations}

\section{Part A}

Calculating P1 and P2

For the calculation of the height of the bed in atmosphere from Table 2 first of all converting the first and last readings of $\mathrm{H} 1$ column in meters as:

$$
\begin{aligned}
& H_{1} 238 m \Rightarrow \frac{238}{1000} \Rightarrow 0.238 m \\
& H_{8} 121 m \Rightarrow \frac{121}{1000} \Rightarrow 0.121 m \\
& \text { Density } \Rightarrow 1000 \mathrm{~kg} / \mathrm{m}^{3} \\
& \text { Gravity } \Rightarrow 9.81
\end{aligned}
$$

Now calculating P1 where the value forH1 above is the same for $\mathrm{Z} 1$, therefore from the formula:

$$
\begin{aligned}
& P \Rightarrow \rho^{*} g * z \\
& P_{1} \Rightarrow 1000 * 9.81 * 0.238 \Leftrightarrow 2334.78 \mathrm{pa}
\end{aligned}
$$


Citation: Aminu MD, Ardo BU (2015) A Novel Approach for Determining Permeability in Porous Media. J Pet Environ Biotechnol 6: 226. doi:10.4172/2157-7463.1000226

Page 3 of 5

\begin{tabular}{|c|c|c|c|c|c|c|c|c|c|c|c|c|}
\hline $\begin{array}{c}Q \\
\left(\mathrm{~cm}^{3} \mathrm{~min}^{-1}\right)\end{array}$ & $Q\left(\mathbf{c m}^{3} \mathbf{s}^{-1}\right)$ & $\mathrm{H} 1\left(\mathrm{mmH}_{2} \mathrm{O}\right)$ & $\mathrm{Z} 1=\mathrm{H} 1(\mathrm{~m})$ & $\mathrm{h} 2\left(\mathrm{mmH}_{2} \mathrm{O}\right)$ & $\mathrm{Z} 2=\mathrm{H} 2(\mathrm{~m})$ & $P_{(\mathrm{H} 1)} \mathbf{p a}$ & $\mathrm{P}(\mathrm{H} 2)$ pa & P1 (atm) & P2 (atm) & $\Delta \mathrm{P}(\mathrm{atm})$ & Temp $\left({ }^{\circ} \mathrm{C}\right)$ & $\begin{array}{c}\mathrm{P} \\
\text { (atms) }\end{array}$ \\
\hline 50 & 0.8333333 & 238 & 0.238 & 257 & 0.257 & 2334.78 & 2521.17 & 0.0230419 & 0.0248814 & 0.001839 & 21.5 & 0.002032 \\
\hline 100 & 1.6666667 & 224 & 0.224 & 274 & 0.274 & 2197.44 & 2687.94 & 0.0216865 & 0.0265273 & 0.004841 & 21.5 & 0.004259 \\
\hline 150 & 2.5 & 210 & 0.21 & 289 & 0.289 & 2060.1 & 2835.09 & 0.0203311 & 0.0279795 & 0.007648 & 21.5 & 0.007065 \\
\hline 200 & 3.3333333 & 195 & 0.195 & 307 & 0.307 & 1912.95 & 3011.67 & 0.0188789 & 0.0297222 & 0.010843 & 21.5 & 0.010646 \\
\hline 250 & 4.1666667 & 180 & 0.18 & 325 & 0.325 & 1765.8 & 3188.25 & 0.0174267 & 0.0314648 & 0.014038 & 21.5 & 0.014034 \\
\hline 300 & 5 & 164 & 0.164 & 344 & 0.344 & 1608.84 & 3374.64 & 0.0158776 & 0.0333043 & 0.017427 & 21.5 & 0.017905 \\
\hline 350 & 6.6666667 & 144 & 0.144 & 367 & 0.367 & 1412.64 & 3600.27 & 0.0139413 & 0.0355311 & 0.02159 & 21.5 & 0.021486 \\
\hline 400 & 7.5 & 121 & 0.121 & 394 & 0.394 & 1187.01 & 3865.14 & 0.0117146 & 0.0381451 & 0.02643 & 21.5 & 0.025164 \\
\hline
\end{tabular}

Table 2: Part A-height of the bed in atmosphere.

Now converting to atmosphere knowing that:

$1 \mathrm{pa}=====9.869 \mathrm{e}^{-6} \mathrm{~atm}$

$2521.17 \mathrm{pa}==\mathrm{x}$

Calculating: $P_{1} \Rightarrow \frac{2334.78 * 9.869 e^{-6}}{1} \Leftrightarrow 0.02304194 \mathrm{~atm}$

Now calculating P8 where the value for $\mathrm{H} 8$ is the same for $\mathrm{z}$, therefore from the formula:

$$
\begin{aligned}
& P \Rightarrow \rho^{*} g^{*} z \\
& P_{8} \Rightarrow 1000 * 9.81 * 0.121 \Leftrightarrow 1187.01 \mathrm{pa}
\end{aligned}
$$

Now again converting to atmosphere:

Calculating: $P_{8} \Rightarrow \frac{1187.01 * 9.869 e^{-6}}{1} \Leftrightarrow 0.0117146 \mathrm{~atm}$

Calculating now the height in atmosphere for $\mathrm{H} 2$ column from Table 3 converting again the first and last readings in meters as:

$$
\begin{aligned}
& H_{1} 257 m m \Rightarrow \frac{257}{1000} \Rightarrow 0.257 m \\
& H_{8} 394 m m \Rightarrow \frac{394}{1000} \Rightarrow 0.394 m
\end{aligned}
$$

Calculating P2 where again the value for $\mathrm{H} 2$ is the same for $\mathrm{z}$, therefore from the formula:

$$
\begin{aligned}
& P \Rightarrow \rho^{*} g * z \\
& P_{1} \Rightarrow 1000 * 9.81 * 0.257 \Leftrightarrow 2521.17 \mathrm{pa}
\end{aligned}
$$

Converting to atmosphere:

Calculating: $P_{1} \Rightarrow \frac{2521.17 * 9.869 e^{-6}}{1} \Leftrightarrow 0.024881427 \mathrm{~atm}$

Again calculating P8 where the value for $\mathrm{H} 8$ is the same for $\mathrm{z}$, therefore from the formula:

$$
\begin{aligned}
& P \Rightarrow \rho^{*} g^{*} z \\
& P_{10} \Rightarrow 1000 * 9.81 * 0.394 \Leftrightarrow 3865.14 \mathrm{pa}
\end{aligned}
$$

Now converting to atmosphere:

Calculating: $P_{8} \Rightarrow \frac{3865.14 * 9.869 e^{-6}}{1} \Leftrightarrow 0.038145067 \mathrm{~atm}$

After for the calculation of the pressure drop $(\Delta \mathrm{P})$ from the formula below:

$$
\begin{aligned}
& \Delta P \Rightarrow P_{2}, H_{2}-P_{1}, H_{1} \\
& \Delta P \Rightarrow 0.024881427-0.02304194 \Rightarrow 0.001839 a \mathrm{tm}
\end{aligned}
$$

After the flow rate in $\left(\mathrm{cm}^{3} / \mathrm{s}\right)$ was obtained as: Q/60 mins
Flow rate $=50\left(\mathrm{~cm}^{3} / \mathrm{min}\right)=50 / 60=0.8333\left(\mathrm{~cm}^{3} / \mathrm{s}\right)$

Flow rate $=400\left(\mathrm{~cm}^{3} / \mathrm{min}\right)=400 / 60=6.66666667\left(\mathrm{~cm}^{3} / \mathrm{s}\right)$

Calculating Area, Gradient and Permeability:

To calculate the gradient, first of all the area of the bed was calculated from the following data readings taken from the experiment:

Diameter $=38 \mathrm{~mm}$

Length $=305 \mathrm{~mm}$

Viscosity of water $=1115.6 \mathrm{cp}(0.89 \mathrm{~Pa} \mathrm{~s})$

1 Darcy $=1 \times 10^{-8} \mathrm{~cm}^{2}$

To convert the taken readings to $\mathrm{cm}$ :

Diameter $38 \mathrm{~mm}=38 / 10=3.8 \mathrm{~m}$

Length $305 \mathrm{~mm}=305 / 10=30.5 \mathrm{~cm}$

Therefore the area was calculated from the following formula from: $\frac{\pi d^{2}}{4}=\pi * \frac{3.8^{2}}{4}=11.34 \mathrm{~cm}^{2}$

Now from the formula below the gradient could be obtained as:

Gradient $=\frac{\mu L}{A K}$

gradient $=\frac{(Q 8-Q 1)}{(\Delta \mathrm{P} 8-\Delta \mathrm{P} 1)}-\frac{(6.66666667-0.8333)}{(0.02643-0.001839)}=237.21433 \mathrm{~cm}^{2}$

Where the slope is equal to gradient:

gradient $=$ slope $=237.21433$

Calculating the permeability $(\mathrm{K})$ from the formula:

$$
K \Rightarrow \frac{m^{*} \mu^{*} L}{A}
$$

Where $\mathrm{m}$ is equal to the slope or gradient of the graph, therefore:

\begin{tabular}{|c|c|c|c|c|c|c|c|}
\hline Case & $\begin{array}{c}\mathbf{Q W ,} \\
\mathbf{c c} / \mathbf{s}\end{array}$ & $\begin{array}{c}\mathbf{Q}, \\
\mathbf{c c . s}\end{array}$ & $\begin{array}{c}\mathbf{A P}, \\
\mathbf{a t m}\end{array}$ & $\mathbf{K w}$ & $\mathbf{K r w}$ & Ko & Kro \\
\hline $\begin{array}{c}80 \% \mathrm{~W}, \\
20 \% \mathrm{O}\end{array}$ & 50 & 2 & 0.03 & 201.84999 & 0.3164063 & 2.4919752 & 0.003906 \\
\hline $\begin{array}{c}60 \% \mathrm{~W}, \\
40 \% \mathrm{O}\end{array}$ & 40 & 3 & 0.05 & 7.8758723 & 0.0123457 & 126.01396 & 0.197531 \\
\hline $\begin{array}{c}40 \% \mathrm{~W}, \\
60 \% \mathrm{O}\end{array}$ & 30 & 4 & 0.06 & 39.871604 & 0.0625 & 3229.5999 & 5.0625 \\
\hline $\begin{array}{c}20 \% \mathrm{~W}, \\
80 \% \mathrm{O}\end{array}$ & 20 & 5 & 0.08 & 51673.598 & 81 & 163314.09 & 256 \\
\hline
\end{tabular}

Table 3: Part B-relative oil permeability. 
Citation: Aminu MD, Ardo BU (2015) A Novel Approach for Determining Permeability in Porous Media. J Pet Environ Biotechnol 6: 226. doi:10.4172/2157-7463.1000226

Page 4 of 5

$$
K \Rightarrow \frac{237.21433 * 1 * 30.5}{0.1134} \Rightarrow 637.9456577 D
$$

\section{Part B}

Converting now the water saturation $\left(\mathrm{S}_{\mathrm{w}}\right)$ :

$S_{W 1} \Rightarrow 80 \% \Leftrightarrow 80 / 100 \Rightarrow 0.8$

$S_{W 1} \Rightarrow 60 \% \Leftrightarrow 60 / 100 \Rightarrow 0.6$

$S_{W 1} \Rightarrow 40 \% \Leftrightarrow 40 / 100 \Rightarrow 0.4$

$S_{W 1} \Rightarrow 20 \% \Leftrightarrow 20 / 100 \Rightarrow 0.2$

Converting the oil saturation $\left(\mathrm{S}_{\mathrm{O}}\right)$ :

$S_{O 1} \Rightarrow 20 \% \Leftrightarrow 20 / 100 \Rightarrow 0.2$

$S_{O 1} \Rightarrow 40 \% \Leftrightarrow 60 / 100 \Rightarrow 0.4$

$S_{O 1} \Rightarrow 60 \% \Leftrightarrow 60 / 100 \Rightarrow 0.6$

$S_{O 1} \Rightarrow 80 \% \Leftrightarrow 80 / 100 \Rightarrow 0.8$

For the calculation of the relative oil permeability $\left(\mathrm{k}_{\mathrm{ro}}\right)$ the formula used was:

$K_{R O} \Rightarrow\left[\frac{1-S_{W}}{1-S_{W C}}\right]^{4}$

Calculating:

$K_{R O} \Rightarrow((1-0.8) /(1-0.2))^{4} \Rightarrow 0.003906$

$K_{R O} \Rightarrow((1-0.6) /(1-0.4))^{4} \Rightarrow 0.197531$

$K_{R O} \Rightarrow((1-0.4) /(1-0.6))^{4} \Rightarrow 5.0625$

$K_{R O} \Rightarrow((1-0.2) /(1-0.8))^{4} \Rightarrow 256$

For the calculation of the relative oil permeability $\left(\mathrm{k}_{\mathrm{rw}}\right)$ the formula used was:

$$
K_{R W} \Rightarrow\left[\frac{S_{W}-S_{W C}}{1-S_{W C}}\right]^{4}
$$

Where the formula could be rearranged as:

$$
\begin{aligned}
& K_{R W} \Rightarrow\left[\frac{S_{W}-S_{O}}{1-S_{O}}\right]^{4} \\
& K_{R W 1} \Rightarrow((0.8-0.2) /(1-0.2))^{4} \Rightarrow 0.3164063 \\
& K_{R W 2} \Rightarrow((1-0.6) /(1-0.4))^{4} \Rightarrow 0.123457 \\
& K_{R W 3} \Rightarrow((0.4-0.6) /(1-0.6))^{4} \Rightarrow 0.0625 \\
& K_{R O} \Rightarrow((0.2-0.8) /(1-0.8))^{4} \Rightarrow 81
\end{aligned}
$$

For the calculation of the oil permeability $\left(\mathrm{K}_{\mathrm{o}}\right)$ could be calculated through the following formula:

$$
K_{R O} \Rightarrow K_{O} / K
$$

Where:

$$
\begin{aligned}
& K \Rightarrow 637.9456577 D \\
& K_{R O} \Rightarrow K_{O} / K \Leftrightarrow K_{O} \Rightarrow K_{R O} * K \\
& K_{O 1} \Rightarrow 0.003906 * 637.9456577 \Rightarrow 2.491975225
\end{aligned}
$$

$$
\begin{aligned}
& K_{O 2} \Rightarrow 0.197531 * 637.9456577 \Rightarrow 126.0139571 \\
& K_{O 2} \Rightarrow 5.0625 * 637.9456577 \Rightarrow 3229.599892 \\
& K_{O 4} \Rightarrow 256 * 637.9456577 \Rightarrow 163314.0884
\end{aligned}
$$

Finally calculating water permeability $\left(\mathrm{K}_{\mathrm{w}}\right)$ using the same formula:

$$
K_{R W} \Rightarrow K_{W} / K
$$

Where again:

$$
\begin{aligned}
& K \Rightarrow 637.9456577 D \\
& K_{R W} \Rightarrow K_{W} / K \Leftrightarrow K_{W} \Rightarrow K_{R W} * K \\
& K_{O 1} \Rightarrow 0.3164063 * 637.9456577 \Rightarrow 201.8499933 \\
& K_{O 2} \Rightarrow 0.123457 * 637.9456577 \Rightarrow 7.875872317 \\
& K_{O 2} \Rightarrow 0.0625 * 637.9456577 \Rightarrow 39.87160361 \\
& K_{O 4} \Rightarrow 81 * 637.9456577 \Rightarrow 51673.59827
\end{aligned}
$$

\section{Discussion}

Following the data obtained in from Table 1, it can be noticed that the pressure drop increases as the flow rate increases across the bed. Increasing the flow rate from 50 to $4003 / \mathrm{min}$, pressure drop values were noted as $(0.001839-0.02643)$. It is therefore true that the pressure drop is proportional to the runoff of the liquid through the bed. When plotting the flow rate against pressure drop (Figure 1), the straight line shows exactly this relationship between these properties. Again it was noticed that the exit water temperature didn't fluctuate profusely, as throughout the experiment, it increased up to 21.5 degrees centigrade. As demonstrated in theoretical knowledge, permeability increases with the rise of temperature due to a decrease of viscosity, therefore this means that if the temperature somewhere at the middle of the run decreases, permeability would in proportion, decrease. In this experiment however, temperature was kept relatively constant throughout the exercise [4]. The recorded value for permeability at 637.9456577 (as is seen in the table below) is equivalent to a very good reservoir in the real life (Figure 2).

Therefore the rock has a very good quality to allow flow of fluids.

Also there could be some inaccuracy of the results, because is very unlikely to get a very good rock properties in the real life, thus, the experiment could have been affected by the entry of some air bubbles that were spotted going through the pipes were-this can affect accuracy of results. A high pressure manometer wasn't used. Also a small fluidizing due to the rise of the sand may affect the increase of the flow rate started later on the experiment.

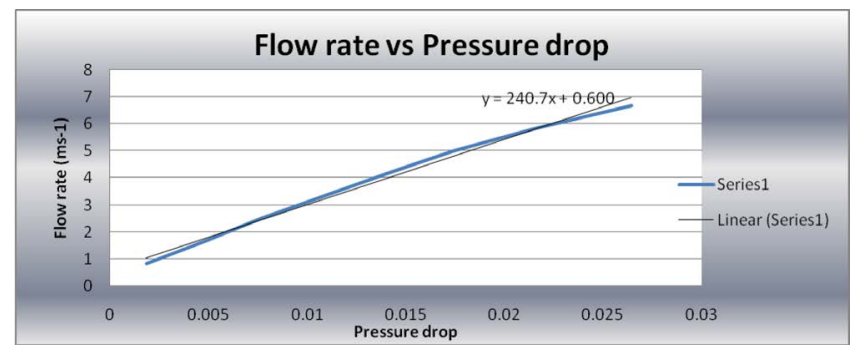

Figure 1: Flow rate vs. pressure drop. 


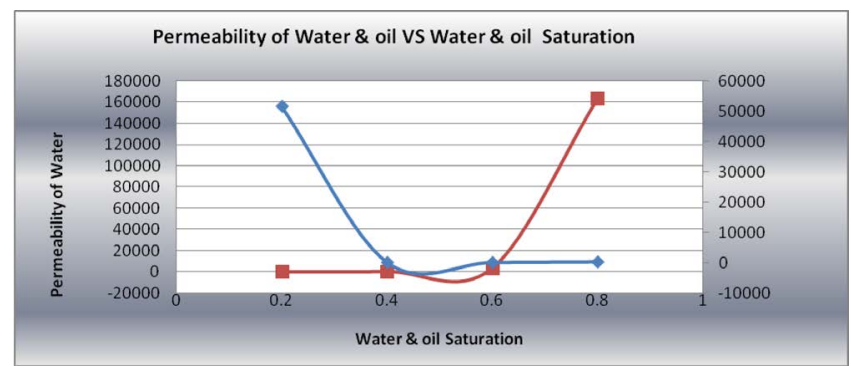

Figure 2: Permeability of water and oil water and oil saturation.

\section{Conclusion}

It can be concluded that the certain rock properties of porosity such as particle size, as well as fluid properties such as viscosity, density, etc affected the final results. For non-compressible liquids or fluids, the permeability of the rock is maintained as same irrespective of the type of fluid that flows through it. For this reason, an experiment like this is very useful in the real life because of the fact that determining the absolute permeability of a reservoir would out-rightly inform us on whether the rock material is good and viable for flow and accumulation of petroleum. The apparatus used for permeability is shown in Figure 3.

It is therefore established in this experiment that the permeability of rock is directly proportional to the permeability of the sand mentioned above in the objective. In this concept, when there is an increase in particle size, there is definitely an increase in permeability and porosity of rock sediment. It should also be highlighted that reservoirs are different due to the inconsistencies in particle sizes, degree of cementation between particles, formation of sediments by individual particles, etc.

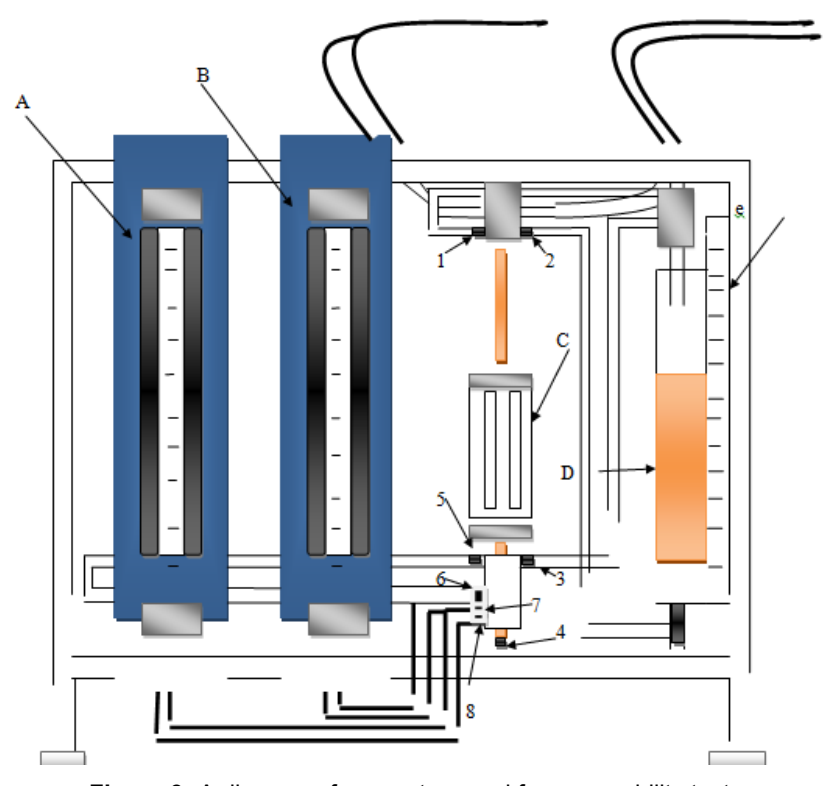

Figure 3: A diagram of apparatus used for permeability test.

\section{References}

1. ASME Shale Shaker Committee (2005) Drilling Fluids Processing. Handbook Gulf Professional, Gulf Professional Publishing

2. Civan F (2007) Reservoir formation damage: fundamentals, modelling, assessment, and mitigation, Houston, TX, USA

3. Darling $T$ (2005) Well logging and formation evaluation, Gulf Drilling Guides Elsevier

4. Jahn F, Cook M, Graham M (2006) Hydrocarbon Exploration and Production, Elsevier 